\title{
A Prospective Longitudinal Brain Morphometry Study of Children with Sickle Cell Disease
}

\author{
R. Chen, M. Arkuszewski, J. Krejza, R.A. Zimmerman, E.H. Herskovits, and E.R. Melhem
}

\begin{abstract}
BACKGROUND AND PURPOSE: Age-related changes in brain morphology are crucial to understanding the neurobiology of sickle cell disease. We hypothesized that the growth trajectories for total GM volume, total WM volume, and regional GM volumes are altered in children with sickle cell disease compared with controls.
\end{abstract}

MATERIALS AND METHODS: We analyzed T7-weighted images of the brains of 28 children with sickle cell disease (mean baseline age, 98 months; female/male ratio, 15:13) and 28 healthy age- and sex-matched controls (mean baseline age, 99 months; female/male ratio, 16:12). The total number of MR imaging examinations was 141 (2-4 for each subject with sickle cell disease, 2-3 for each control subject). Total GM volume, total WM volume, and regional GM volumes were measured by using an automated method. We used the multilevel-model-forchange approach to model growth trajectories.

RESULTS: Total GM volume in subjects with sickle cell disease decreased linearly at a rate of $411 \mathrm{~mm}^{3}$ per month. For controls, the trajectory of total GM volume was quadratic; we did not observe a significant linear decline. For subjects with sickle cell disease, we found 35 brain structures that demonstrated age-related GM volume reduction. Total WM volume in subjects with sickle cell disease increased at a rate of $452 \mathrm{~mm}^{3}$ per month, while the trajectory of controls was quadratic.

CONCLUSIONS: There was a significant age-related decrease in total GM volume in children with sickle cell disease. The GM volume reduction was spatially distributed widely across the brain, primarily in the frontal, parietal, and occipital lobes. Total WM volume in subjects with sickle cell disease increased at a lower rate than for controls.

ABBREVIATIONS: NIHPD = National Institutes of Health MRI Study of Normal Brain Development; SCD $=$ sickle cell disease

$\mathbf{S}$ ickle cell disease (SCD) is one of the most common severe single-gene disorders in the United States. ${ }^{1}$ SCD is a hemolytic disease characterized by abnormally shaped (sickled) red blood cells, leading to anemia. ${ }^{2}$ Patients with SCD have at least

\footnotetext{
Received May 14, 2014; accepted after revision July 16.

From the Department of Diagnostic Radiology and Nuclear Medicine (R.C., J.K., E.H.H., E.R.M.), University of Maryland, Baltimore, Maryland; Department of Neurology (M.A.), Medical University of Silesia, Katowice, Poland; Department of Radiology (R.A.Z.), Children's Hospital of Philadelphia, Philadelphia, Pennsylvania; and Department of Radiology (R.C., R.A.Z.), Raymond and Ruth Perelman School of Medicine, University of Pennsylvania, Philadelphia, Pennsylvania.

Some data used in the preparation of this article were obtained from the Pediatric MRI Data Repository created by the National Institutes of Health MRI Study of Normal Brain Development. This is a multisite, longitudinal study of typically developing children, from ages newborn through young adulthood, conducted by the Brain Development Cooperative Group and supported by the National Institute of Child Health and Human Development, the National Institute on Drug Abuse, the National Institute of Mental Health, and the $\mathrm{Na}$ tional Institute of Neurological Disorders and Stroke (contract numbers N01HD02-3343, N01-MH9-0002, and N01-NS-9-2314, -2315, -2316, -2317, -2319 and -2320).

This article reflects the views of the authors and may not reflect the opinions or views of the National Institutes of Health.
}

1 sickle cell hemoglobin chain and 1 other abnormal $\beta$ globin chain. The most common type of SCD is sickle cell anemia $(60 \%-70 \%$ of cases of SCD), characterized by 2 sickle cell hemoglobin genes. The pathophysiology of SCD includes hemoglobin polymerization, chronic anemia, hemolysis, and vasculopathy.

MR imaging provides in vivo structural or functional images of the brain. MR-based brain morphometry centers on neuroanatomic changes. It could provide a method for demonstrating

\footnotetext{
Rong Chen and Edward H. Herskovits were supported by National Institutes of Health grant R01 AG13743, R03 EB-009310 and the American Recovery and Reinvestment Act. Rong Chen was supported by an Institute for Translational Medicine and Therapeutics fellowship, University of Pennsylvania. Jaroslaw Krejza, Michal Arkuszewski, and Elias R. Melhem were supported by National Institutes of Health grant R01 NS 046717

Please address correspondence to Rong Chen, PhD, MTR, Department of Diagnostic Radiology and Nuclear Medicine, University of Maryland, 22 South Greene St, Baltimore, MD 21201; e-mail: rchen@umm.edu

- Indicates open access to non-subscribers at www.ajnr.org

三 Indicates article with supplemental on-line table.

http://dx.doi.org/10.3174/ajnr.A4101
} 

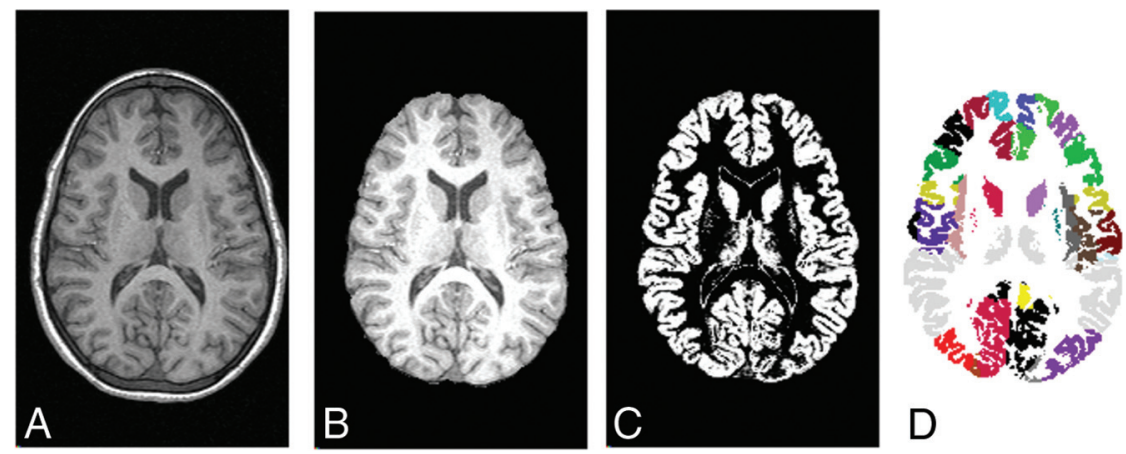

children with SCD. Inclusion criteria were the following: 1) sickle cell anemia genotype: homozygous SS, confirmed by isoelectric focusing with DNA-based confirmatory testing or parental studies, 2) no deficits on neurologic examination, 3 ) no history of stroke, and 4) transcranial Doppler sonography mean velocities $<170 \mathrm{~cm} / \mathrm{s}$ in a routine screening examination. Exclusion criteria were the following: 1) a history of major head injury requiring a visit to an emergency department; 2) a history of seizure disorder requiring anticonvulsant therapy; 3 ) chronic transfusion therapy; 4) occur-

brain injury in children with SCD, complementary to lesionbased analysis. Brain atrophy is defined as the abnormal loss of brain-tissue volume and manifests visually as lobar asymmetry, open sulci, or ventricular dilation. ${ }^{3}$ A quantitative approach to detecting atrophy is brain morphometry, which provides quantitative measurements of brain-tissue volumes. There have been several studies of SCD based on brain morphometry. ${ }^{4-7}$ Baldeweg et $\mathrm{al}^{4}$ performed whole-brain voxel-based morphometric analysis on 36 children and adolescents with SCD (9-24 years of age) and 31 controls ( $8-25$ years of age). They found that in comparison with controls, subjects with SCD (with or without silent infarcts) demonstrated white matter density reduction and gray matter density changes. Kirk et $\mathrm{al}^{6}$ used surface-based morphometry to examine cortical thinning in children with SCD.

Studies have assessed age-related changes in neurocognitive functioning in children with SCD. ${ }^{8}$ In a longitudinal study of 373 children with SCD (6-18 years of age), Wang et $\mathrm{al}^{8}$ reported that the scores for verbal intelligence quotient, math achievement, and coding declined with increasing age.

Age-related changes in brain morphology are crucial to understanding the neurobiology of SCD. However, most previous morphometric studies have been only cross-sectional. No previous studies, to our knowledge, have assessed longitudinal MR-based morphometric changes in children with SCD. We addressed the problem of characterizing brain structure development in children with SCD in this study. We hypothesized that growth trajectories for total gray matter volume, total white matter volume, and regional gray matter volumes (normalized to total brain volume) are altered in children with SCD compared with controls. We tested this hypothesis on the basis of data from a prospective longitudinal study of children with SCD.

\section{MATERIALS AND METHODS \\ Participants}

This study included children with SCD and healthy children. The SCD population was drawn from the Comprehensive Sickle Cell Center at the Children's Hospital of Philadelphia. The institutional review board of the Children's Hospital of Philadelphia approved the protocol of this prospective study, which was also compliant with the Health Insurance Portability and Accountability Act. Written informed consent was given by subjects' parents (with assent for subjects 7 years and older). The SCD group had 28 rence of a sickle cell anemia pain episode, acute chest syndrome, or another significant medical problem in the period of laboratory blood testing, neuropsychological testing, and sonographic studies; 5) a history of prenatal or perinatal hypoxic-ischemic brain injury; and 6) evidence of HIV infection. The SCD group was selected from a larger cohort of 67 children with SCD without prior clinically overt stroke or transient ischemic attack. ${ }^{9}$ The subjects with SCD in the current study had longitudinal MR imaging examinations.

The control group included 28 healthy children selected from the National Institutes of Health MRI Study of Normal Brain Development (NIHPD). ${ }^{10}$ Subjects were excluded if they met criteria "established or highly suspected to adversely impact healthy brain development." Informed consent was obtained from parents and adolescents, and assent was obtained from children. Protocols and procedures were approved by relevant institutional review boards. For each subject in the SCD group, we selected an age- and sex-matched child from the NIHPD data base. If there was no age- and sex-matched child in the NIHPD data base for a participant in the SCD group, we selected a healthy child whose age was close to that of the subject with SCD.

\section{MR Imaging and Analysis}

The 28 children in the SCD group were those who had a minimum of 2 yearly brain MR imaging examinations, including a baseline examination. All subjects with SCD underwent MR imaging by using a 3T Magnetom Trio scanner (Siemens, Erlangen, Germany) at the Children's Hospital of Philadelphia. The T1weighted MR imaging parameters were TR/TE/TI, 1380/2.15/800 ms; matrix, $256 \times 256$; voxel size, $1 \times 1 \times 1 \mathrm{~mm}$. Among these 28 subjects, 16 children had 2 MR imaging examinations, 9 children had 3 , and 3 children had 4 . The total number of MR imaging examinations in this study was 71 .

For the control group, a whole-brain 3D T1-weighted spoiled gradient recalled-echo sequence was acquired. The matrix was $256 \times 256$, and section thicknesses were 1 and $1.4-1.8 \mathrm{~mm}$ on the Siemens and GE scanners, respectively; MR image acquisition details are reported in the Brain Development Cooperative Group reference. ${ }^{11}$ Each child in the control group underwent 2-3 biannual MR imaging examinations.

The MR image analysis procedures (Fig 1) were as follows: First, we used the FMRIB Brain Extraction Tool from FSL (http:// 


\begin{tabular}{lccc}
\hline & SCD & Controls & $\boldsymbol{P}$ Value \\
\hline Mean age across all observations (mo) & 113 & 116 & .732 \\
Age range across all observations (mo) & $33-190$ & $31-194$ & NA \\
Mean age at baseline (mo) & $98 \pm 37.6$ & $99 \pm 38.0$ & .947 \\
Age range at baseline (mo) & $33-156$ & $31-157$ & NA \\
Sex (female/male) & $15 / 13$ & $16 / 12$ & .788 \\
\hline
\end{tabular}

Note:-NA indicates not applicable.

www.fmrib.ox.ac.uk/fsl $)^{12}$ to exclude nonbrain tissues. Then we used SPM5 (Wellcome Department of Imaging Neuroscience, London, United Kingdom $)^{13}$ to perform joint segmentation and registration, yielding GM, WM, and CSF by using labeled voxels, normalized to the standard Montreal Neurological Institute space. Automatic volume calculation was performed by using a software package called Individual Brain Atlases Using Statistical Parametric Mapping Software (http://www.thomaskoenig.ch/ Lester/ibaspm.htm). We thus parcellated each individual brain into 90 brain structures defined in the brain template of Automated Anatomical Labeling (AAL). ${ }^{14}$ On the basis of the results of tissue segmentation and brain parcellation, we calculated total GM volume, total WM volume, total brain volume (defined as the sum of GM and WM volumes), and regional GM volumes for the 90 Automated Anatomical Labeling structures, measured in cubic millimeters. For regional GM volumes, we were interested in regional rather than global effects; therefore, we adjusted regional GM volumes for each subject's total brain volume in the same MR image. For a brain structure with index $k$, we defined relative volume as $10,000 \times\left[V_{k} /(\right.$ total brain volume $\left.)\right]$, where $V_{k}$ is the raw regional volume of structure $k$; we multiplied the fraction $V_{k}$ l (total brain volume) by 10,000 because $V_{k} /$ (total brain volume) was typically a very small number and not stable under numeric procedures. These regional volumes are relative measurements and dimensionless. This image-analysis pipeline had been used in other studies of pediatric populations. ${ }^{15-18}$

\section{Statistical Analysis: Modeling Trajectories}

To analyze the trajectory of brain growth (for either children with SCD or for healthy children in the control group), we used the multilevel-model-for-change ${ }^{19}$ approach, which addresses simultaneously within-subject and between-subject questions about change rates. This method can handle varying numbers of measurements and variably spaced measurements. We used the $x t$ mixed procedure in STATA 11 (StataCorp, College Station, Texas) to implement this analysis.

There are 2 components in the multilevel model for change: level 1 and level 2 submodels. The level 1 component (the individual growth model) represents the change that each member undergoes during the time under study. If one adopts an individual growth model in which change is a linear function of age, the level 1 model is

$$
Y_{i j}=\pi_{0 i}+\pi_{1 i} \times A g e+\varepsilon_{i j}
$$

where $Y_{i j}$ is the outcome variable of interest, such as total GM volume for subject $i$ at time $j, \pi_{0 i}$ is the intercept of the growth curve, $\pi_{1 i}$ is the slope (rate of change) of the growth curve, and $\varepsilon_{i j}$ is the random measurement error. We recentered Age so that its mean was 9 years (108 months, the sample mean) to ensure that the value of Age associated with $\pi_{0 i}$ was within the observed range of Age. ${ }^{19}$

The level 2 model allows the value of each individual's growth parameters $\left(\pi_{0 i}\right.$ and $\pi_{1 i}$ ) to be distributed about the relevant population average. The level 2 model is

$$
\begin{aligned}
& \pi_{0 i}=\gamma_{00}+\zeta_{0 i} \\
& \pi_{1 i}=\gamma_{10}+\zeta_{1 i},
\end{aligned}
$$

where $\gamma_{00}$ and $\gamma_{10}$ represent the population average initial status and rate of change, respectively, and $\zeta_{0 i}$ and $\zeta_{1 i}$ are level 2 residuals. We assume that $\varepsilon_{i j} \sim N\left(0, \sigma_{\varepsilon}{ }^{2}\right)$ and $\zeta_{0 i}$ and $\zeta_{1 i}$ follow a joint Gaussian distribution with the mean zero and the covariance matrix $\Sigma$. We used the maximum-likelihood estimation procedure in STATA to estimate model parameters.

Sometimes the growth trajectory is quadratic. For this case, we added $A g e^{2}$ to the level 1 model. For a quadratic change trajectory, the level 1 model is

$$
Y_{i j}=\pi_{0 i}+\left(\pi_{1 i} \times A g e\right)+\left(\pi_{2 i} \times A g e^{2}\right)+\varepsilon_{i j},
$$

where $\pi_{0 i}$ is the intercept of the growth curve, $\pi_{1 i}$ is the instantaneous rate of change, and $\pi_{2 i}$ is curvature. Note that $\pi_{1 i}$ in the linear change and quadratic change trajectories have different interpretations. We selected a suitable level 1 model (linear change or quadratic change) on the basis of the Bayesian information criterion. ${ }^{19}$

We constructed the multilevel models for changes (total GM and WM volume) for the SCD and control groups, respectively. If the growth curve was linear, our null hypothesis was that $\gamma_{10}$, the slope of the population-change trajectory, is zero. If the growth curve was quadratic, our null hypothesis was that $\gamma_{10}$, the instantaneous rate of change, is zero.

We constructed multilevel models for change for the 90 regional GM volumes for the SCD group to test whether $\gamma_{10}$ is zero. We performed Bonferroni multiple-comparison correction; if the adjusted $P$ value after multiple-comparison correction was $<.05$, we rejected the corresponding null hypothesis.

\section{RESULTS}

The demographic characteristics of the study are summarized in the Table. For the SCD group, each child underwent 2-4 annual examinations. At baseline, the mean age was $98 \pm 37.6$ months. The female/male ratio was 15:13. For the control group, each child underwent 2-3 biannual examinations. At baseline, the mean age was $99 \pm 38.0$ months; the female/male ratio was 16:12. There were no significant differences in the baseline age $(P=.947$ based on the 2 -sample $t$ test $)$ or female/male ratio $(P=.788$ based on the $\chi^{2}$ test $)$ and the mean age across all observations $(P=.732$ based on the 2-sample $t$ test). There were no significant differences in the age range across all observations and the age range at baseline.

We did not observe any neurologic clinical events such as a stroke or TIA during the period of observation in our patients with SCD. We did not find any Moyamoya disease syndrome in children with SCD.

AJNR Am J Neuroradiol 36:403-10 Feb 2015 www.ajnr.org 405 

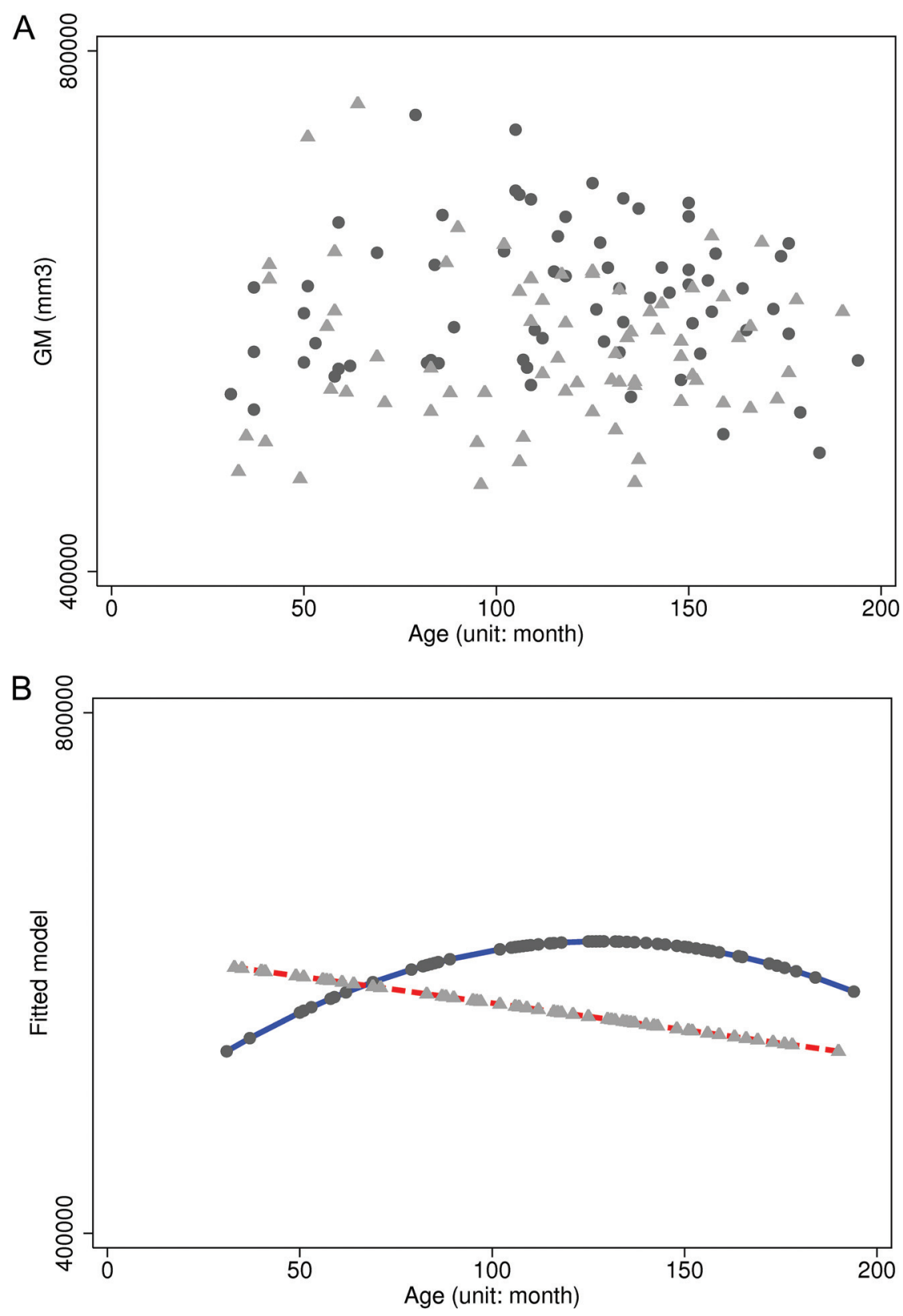

FIG 2. The population growth trajectory for total GM volume. A, the scatterplot of GM volumes versus age. Triangles represent data points for the SCD group, and circles represent data points for healthy control subjects. $B$, the fitted model. Red and blue curves are for the SCD and control groups, respectively.

All MR imaging and MRA examinations were analyzed by 2 experienced neuroradiologists (with 20 and 30 years of experience) blinded to patient data. Discrepancies were resolved by consensus. Silent infarct was defined as an area of abnormal hyperintensity on FLAIR images $\geq 3 \mathrm{~mm}$ in diameter and visible in at least 2 perpendicular planes.

In the first MRA examination, we found 3 stenoses in 2 children, including 1 child with isolated left terminal internal carotid artery (tICA) stenosis (25\%-49\%) and 1 child with mirror stenoses of both anterior cerebral arteries (25\%-49\%).

On the basis of MR imaging data, we found silent infarcts in 15 children, including 1 with stenosis of the left tICA. In 9 children, silent infarcts were observed in the first MR imaging examination (the baseline) and in all following MR imaging examinations; whereas in the remaining 6 children, silent infarcts were recorded only in the follow-up studies. We did not observe significant changes in the total volume of silent infarcts. The median values of the volume of silent infarcts in the following years (years 1-4) were $29.5,33,54$, and $32 \mathrm{~mm}^{3}$, respectively. The small number of subjects with silent infarcts did not allow us to perform trajectory analysis because of a lack of statistical power.

\section{Total GM and WM Volumes}

Figure 2 displays the multilevel model for change for total GM volume. For the SCD group, the best-fitting model of age-related changes in total GM was linear. The intercept $\gamma_{00}$, which was the volume at 9 years of age, was $573,602 \mathrm{~mm}^{3}$. The slope of the population change trajectory, $\gamma_{10}$, was -411 ; that is, total GM volume decreased at a rate of $411 \mathrm{~mm}^{3}$ per month. We rejected the null hypothesis $\left(\gamma_{10}=0\right)$ with $P=.014$. For the control group, the best-fitting model of age-related changes in total GM was quadratic. The intercept (the volume at 9 years of age), the instantaneous rate of change, and curvature were $620,691 \mathrm{~mm}^{3}(P<.001), 362(P=.001)$, and $-8.94(P<.001)$, respectively. The trajectory was $-8.94 A g e^{2}+362 A g e+$ 620,691 . The average rate of change was $272 \mathrm{~mm}^{3}$ per month.

Figure 3 shows the multilevel model for change for total WM volume. For the SCD group, the best-fitting model of agerelated changes in total WM was linear. The intercept $\gamma_{00}$ was $363,260 \mathrm{~mm}^{3}(P<$ $.001)$. The slope of the population change trajectory, $\gamma_{10}$, was $452(P<.001)$; that is, total WM volume grew at a rate of 452 $\mathrm{mm}^{3}$ per month. For the control group, the best-fitting model of age-related changes in total WM was quadratic. The intercept (the volume at 9 years of age), the instantaneous rate of change, and curvature were $423,073 \mathrm{~mm}^{3}(P<.001), 1020(P<.001)$, and -4.37 $(P<.001)$, respectively. The trajectory was $-4.37 \mathrm{Age}^{2}+$ 1020 Age $+423,073$. The average rate of change was $976.3 \mathrm{~mm}^{3}$ per month.

\section{Regional Volumes}

The On-line Table summarizes results for the 90 regional volumes. Because the multilevel models for changes in the On-line Table are for relative regional volumes, the absolute value of rate of change does not have a clear interpretation. Most of brain structures in children with SCD underwent volume reduction (ie, 


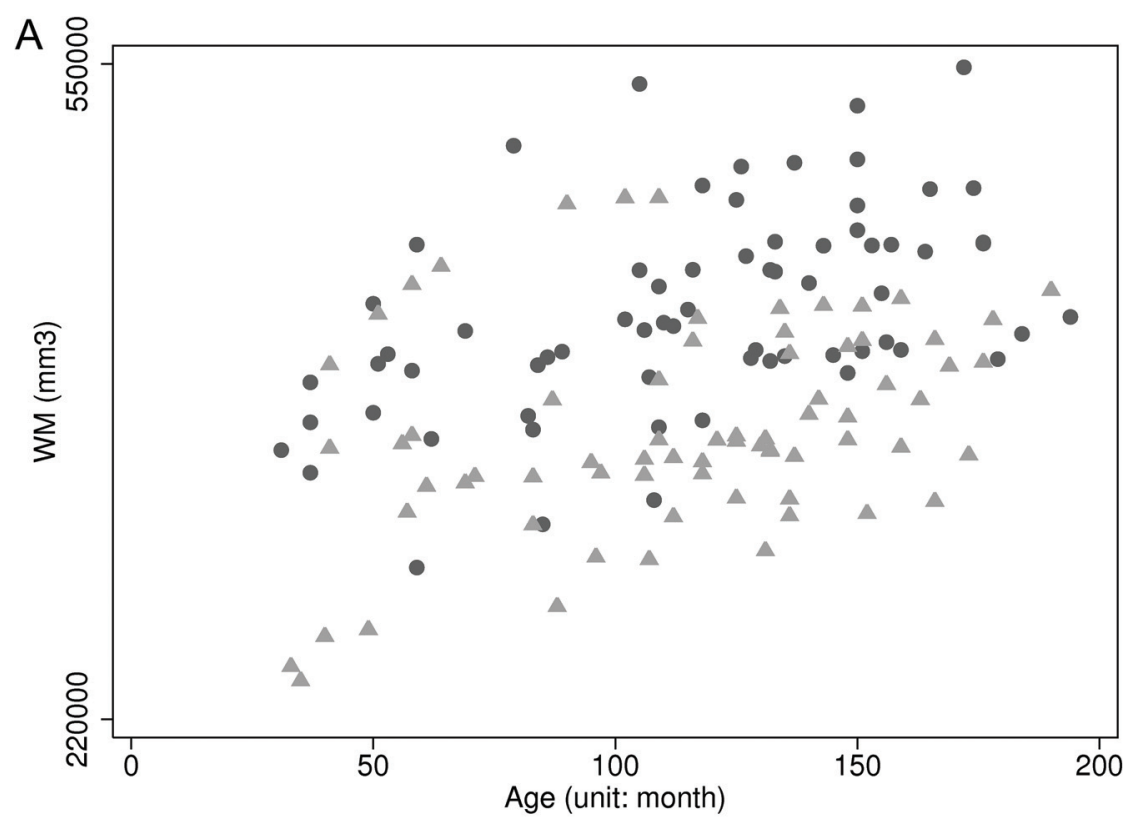

B

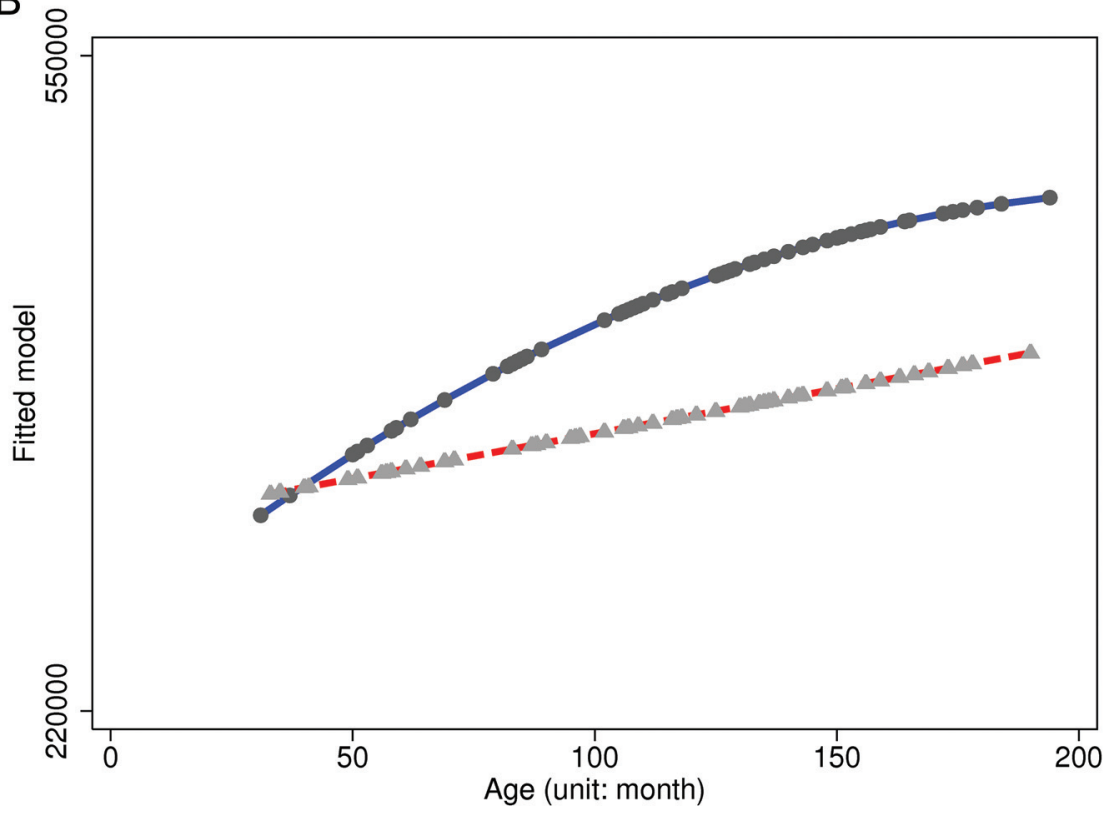

FIG 3. The population growth trajectory for total WM volume. $A$, the scatterplot of WM volumes versus age. Triangles represent data points for the SCD group, and circles represent data points for healthy control subjects. $B$, the fitted model. Red and blue curves are for the SCD and control groups, respectively.

negative slope); only 8 of 90 structures had a positive slope. After performing Bonferroni correction, we found that 35 structures had a nonzero slope ( $P$ value with the Bonferroni correction $<.05)$.

Figure 4 shows slopes for these brain regions. Of note, slopes were negative for all of these structures; that is, these structures underwent volume reduction. The frontal, parietal, and occipital lobes demonstrated significant age-related volume reduction: 11 of 30 structures in the frontal lobes, 9 of 14 structures in the parietal lobes, and 8 of 14 structures in the occipital lobes had nonzero slopes. The temporal lobe and the limbic system demonstrated less age-related volume reduction: One of 12 struc- tures in the limbic system and 4 of 12 structures in the temporal lobes had nonzero slopes.

\section{DISCUSSION}

Atrophy, stroke, and cognitive impairment are major consequences of SCD on the brain. Children with SCD have a high risk for a range of effects on the brain, including overt stroke, cerebral infarcts that occur without overt symptoms of stroke (silent infarct), and insufficient oxygen or glucose delivery or both that result in deficits in brain function without structural tissue damage. Infarct (both overt and silent) and atrophy are common MR imaging findings in children with SCD. ${ }^{20}$

In this study, we found that the total GM trajectories for the SCD group and the control subjects group were different. The trajectory of total GM volume was linear: Total GM volumes decreased at a rate of $411 \mathrm{~mm}^{3}$ per month for children with SCD. For the age- and sex-matched control subjects, the trajectory of total GM volume was quadratic. As shown in Fig 2, total GM volume first increased, peaked at 11 years of age, and then decreased. We did not observe a significant linear decline in total GM volumes. Several MR imaging-based studies describe anatomic brain changes in healthy children and adolescents. ${ }^{21-24}$ They found that total GM volume increases or stabilizes in healthy children as they develop from 3 to 15 years of age. For example, Matsuzawa et $\mathrm{al}^{22}$ reported that total GM volume gradually increased from 2 to 10 years of age. In a study of 35 normally developing children and adolescents between 7 and 16 years of age, Sowell et $\mathrm{al}^{23}$ found that there was no significant change in absolute total GM volume. In contrast, we found a significant decline in total GM volume for children with SCD between 3 and 16 years of age.

For the SCD group, we found 35 Automated Anatomical Labeling atlas structures demonstrated volume reduction with age; these structures were distributed across the entire brain, primarily in the frontal, parietal, and occipital lobes (Figure 4). In contrast, GM volumes of structures in the temporal lobes and limbic system did not change significantly.

The 2 events that occur in brain development for children between 3 and 16 years of age are synaptic pruning and myelination. ${ }^{24}$ Both processes could result in the appearance of GM volume reduction. Synaptic pruning selectively prunes and refines 


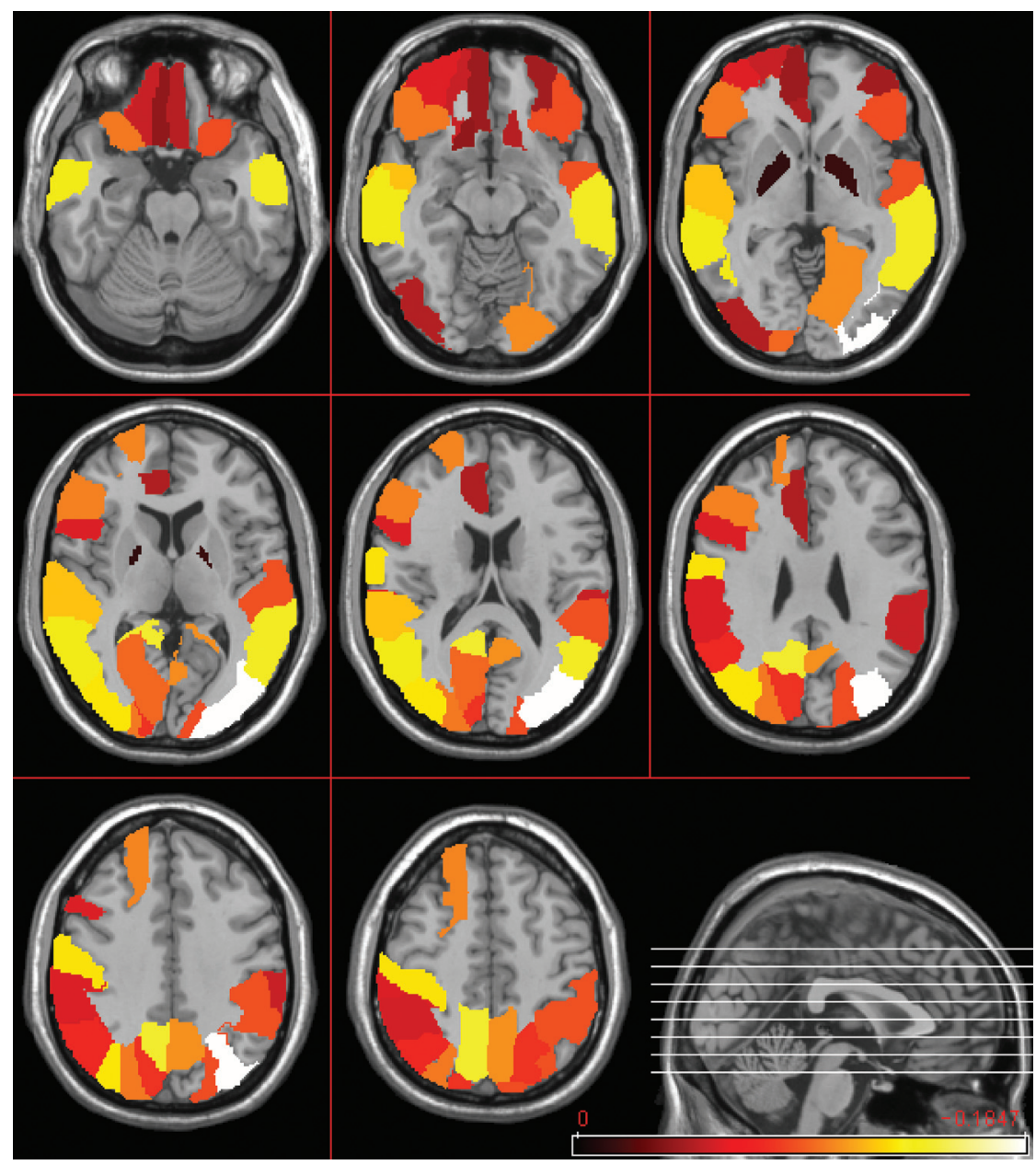

FIG 4. The slopes of the GM growth trajectories for different brain regions. Only regions with an adjusted $P$ value $<.05$ are shown. Brighter color represents more severe volume reduction rate (red indicates less severe volume reduction; white, more severe volume reduction).

In this longitudinal study, we found that the total GM and WM trajectories for the SCD group were different from those of controls. Other cross-sectional morphometric studies also suggested that brain morphologic features were changed in children with SCD. Baldeweg et $\mathrm{al}^{4}$ performed voxel-based morphometric analysis in a study including 36 children and adolescents with SCD (9-24 years of age) and 31 controls ( $8-25$ years of age). They found that in comparison with controls, subjects with SCD (with or without silent infarcts) demonstrated WM density reduction. For subjects with silent infarcts, decreases in WM density were found along the ventricles bilaterally from the anterior frontal to parieto-occipital white matter. For subjects without silent infarcts, the pattern of WM decrease was similar to that for subjects with silent infarcts. Baldeweg et $\mathrm{al}^{4}$ also reported GM changes. For subjects with silent infarcts, areas with GM density decrease were found along the medial wall of the frontal and parietal lobes and areas with GM density decrease were found along the medial frontal surface. In a cross-sectional study including 83 children with SCD and 43 controls, Steen et al ${ }^{5}$ found that patients had $9 \%$ less GM than controls at 5.0 years of age and $5 \%$ less GM than controls at age 9.5 years. Kirk et $\mathrm{al}^{6}$ used surface-based morphometry to examine cortical thinning in children with SCD. They compared regional cortical thickness between the SCD group (12 sub-

synapses that are components of GM. Nonmyelinated peripheral axonal and dendritic fibers do not have normal WM signal on T1-weighted MR images and may be volume-averaged with gray matter. Therefore, myelination could cause an apparent reduction in GM volume. ${ }^{25}$ For healthy children 3-15 years of age, the interaction of synaptic formation, synaptic pruning, and myelination $^{24,26}$ results in a pattern of total GM volume increase or stabilization. ${ }^{22-24}$ In contrast, we found that total GM volume decreases in children with SCD between 3 and 16 years of age and that this GM reduction is distributed across the brain, primarily in the frontal, parietal, and occipital lobes. Alternations in any of these developmental processes (synaptic formation, new cell generation, synaptic pruning, and myelination) could result in apparent or actual GM volume loss.

Many MR imaging-based studies have examined anatomic brain changes in healthy children. ${ }^{21,23}$ Giedd et $\mathrm{al}^{21}$ reported that total WM volume increased linearly with age in 145 healthy children and adolescents; the net increase across the age range of 4-22 years was $12.4 \%$. Our findings are consistent with these results. Furthermore, we found that total WM volume increased in subjects with SCD at approximately half the rate of that seen in control subjects. This finding suggests that WM development is affected by SCD pathology. jects; mean age, 14.6 years) and the control group ( 15 subjects; mean age, 15.6 years) and found that the SCD group had significant bilateral thinning of the medial cortex, including the superior frontal regions, the precuneus, and the paracentral. The frontal areas were among the regions that showed significant thinning.

A possible explanation of the global effect of SCD on brain development is related to the abnormal hemoglobin, which has reduced oxygen affinity and low solubility in its deoxygenated form, which subsequently leads to aggregation and "sickling" of red blood cells. ${ }^{27,28}$ This process is at the core of the disease because sickled cells have greater stiffness, thereby becoming entangled when passing through capillaries and resulting in vaso-occlusive crises observed in this population. ${ }^{29}$ In turn, sickled cells are prematurely destroyed (hemolysis), the consequence of which is anemia. The brains in these children, however, can be normally oxygenated due to a substantial compensatory increase in cerebral blood flow. $^{30}$ This increase in CBF, however, is still controversial because a few recent studies did not detect increased brain perfusion because of substantial compensatory peripheral vasodilation (perfusion paradox). ${ }^{31,32}$

In anemia, oxygen transport is optimized at lower hematocrit levels and higher blood volume to counterbalance abnormal 
rheologic behavior of less pliable sickle red blood cells and the high viscosity of blood, especially saturated with deoxygenated hemoglobin SS. ${ }^{27}$ In a model, ${ }^{33}$ oxygen diffusivity of the capillary bed could be adjusted with respect to perfusion, oxygen partial pressure, hematocrit, and level of vasodilation, thus playing a crucial role in regulating cerebral oxygen delivery in vivo. The autoregulation in children with SCD can also be affected via the alternate hemolytic pathway that begins with intravascular destruction of red blood cells and the release of free hemoglobin and arginase into the plasma. Free hemoglobin binds and inactivates nitric oxide, and concomitantly, free arginase converts L-arginine into ornithine, a nitric oxide substrate, reducing the production of nitric oxide. ${ }^{34}$ Reduced levels of nitric oxide cause vasoconstriction and may interfere with vasodilation that is required as a response to an increase in wall shear stress and other stimuli. Severe hemodynamic disturbances during a period of dynamic brain development might lead to chronic ischemic brain injury and subsequent reduction of GM and WM volume.

We found that the GM volume reduction in children with SCD was primarily in the frontal, parietal, and occipital lobes, while the temporal lobes and limbic system did not change significantly. The exact process underlying this region-specific GM volume reduction in children with SCD is unknown. We speculate that it may be related to a region-specific manner of brain GM maturation. In normal brain development, GM maturation begins first in the dorsal parietal cortices and the spreads over the frontal lobe and caudally and laterally over the parietal and occipital lobes; finally, it spreads over the temporal lobe. ${ }^{35}$ Therefore, we speculate that the frontal, parietal, and occipital lobes demonstrated more reduction in children with SCD because the maturation process in these lobes starts earlier and the accumulated effect of hemodynamic disturbances is more significant.

Recently, there have been several cross-sectional DTI studies of SCD. ${ }^{36,37}$ DTI uses MR imaging in different diffusion-sensitizing gradient directions. It is capable of detecting microstructural WM changes. ${ }^{38}$ Balci et al ${ }^{36}$ analyzed DTI data for 16 patients with SCD (mean age, $25.4 \pm 11.1$ years) and 14 age- and sex-matched healthy controls. They calculated fractional anisotropy and apparent diffusion coefficients for predefined regions of interest. Wide-ranging, bilateral changes in fractional anisotropy values were observed in patients with SCD. Significant reduced fractional anisotropy values, increased ADCs, or both were clustered in several brain regions, including the corpus callosum, frontal white matter, and centrum semiovale. Sun et $\mathrm{al}^{37}$ analyzed DTI data for patients with SCD with no visible lesions (mean age, $15.19 \pm 1.55$ years), patients with SCD with mild gliosis (mean age, $15.38 \pm 3.05$ years), and age-matched controls. They performed tract-based spatial statistics analysis and found that both SCD populations exhibited reduced anisotropy and increased diffusivity compared with controls in several brain regions, including the corpus callosum and centrum semiovale. These 2 crosssectional DTI studies suggest that WM is changed in patients with SCD. This finding is consistent with the results of our study.

In this study, we modeled neurodevelopmental trajectories in children with SCD. We found that trajectories of total GM and WM in children with SCD were different from those of healthy controls. In a longitudinal study of 373 children with SCD, Wang et $\mathrm{al}^{8}$ reported that scores for verbal intelligence quotient, math achievement, and coding declined with increasing age. In a crosssectional study, Chen et $\mathrm{al}^{7}$ found a linear association between intelligence quotient and regional GM volume in children with SCD. This finding suggests that some variance in intelligence quotient in children with SCD is accounted for by regional variability of GM volume.

One of the limitations of this study is that T1-weighted MR images provide little information about which processes contribute to abnormal GM development. Multimodality imaging could partially solve this problem. For example, DTI could provide additional information about myelination (eg, on the basis of diffusion anisotropy). Another limitation is that we did not monitor continuously flow in small vessels during the period of observation. Therefore, we cannot determine the frequency and extent of focal brain lesions corresponding to small-vessel ischemia.

Our future work will focus on the following: 1) determining whether age-related changes in brain morphology are associated with changes in neurocognitive functioning with time. We hope to determine the extent to which variability in neurocognitive functioning can be explained by age-related brain anatomic changes. 2) Using longitudinal DTI to examine white matter development in children with SCD could provide additional information about white matter integrity and myelination.

\section{CONCLUSIONS}

To our knowledge, ours is the first longitudinal morphometry study to investigate brain development in children with SCD. Using a multilevel-model-for-change approach to investigate brain development of children with SCD, we found a significant decline in total GM volume for children with SCD between 3 and 16 years of age. This GM volume reduction is distributed broadly across the brain, primarily in the frontal, parietal, and occipital lobes, largely sparing the limbic system and temporal lobes. The findings of this study may provide valuable information about the neuropathology of SCD.

Our longitudinal morphometric study supports the notion that reduced cerebral GM and WM volumes in neurologically intact children with SCD can be an effect of chronic hemodynamic complications and subsequent insufficient oxygen delivery to neuronal tissue. Early detection of alterations in the delivery and metabolism of oxygen is important because blood transfusion or hydroxyurea therapy can reverse these functional deficits before the structural changes become evident.

Disclosures: Rong Chen—RELATED: Grant: National Institutes of Health. Edward H. Herskovits-UNRELATED: Expert Testimony: medicolegal; Grants/Grants Pending: Center for Health-Related Informatics and Bioimaging.

\section{REFERENCES}

1. Rees DC, Williams TN, Gladwin MT. Sickle-cell disease. Lancet 2010;376:2018-31

2. Bunn HF. Pathogenesis and treatment of sickle cell disease. $N$ Engl J Med 1997;337:762-69

3. Steen RG, Emudianughe T, Hankins GM, et al. Brain imaging findings in pediatric patients with sickle cell disease. Radiology 2003;228:216-25

4. Baldeweg T, Hogan AM, Saunders DE, et al. Detecting white matter injury in sickle cell disease using voxel-based morphometry. Ann Neurol 2006;59:662-72

5. Steen RG, Emudianughe T, Hunte M, et al. Brain volume in pediatric AJNR Am J Neuroradiol 36:403-10 Feb 2015 www.ajnr.org 409 
patients with sickle cell disease: evidence of volumetric growth delay? AJNR Am J Neuroradiol 2005;26:455-62

6. Kirk GR, Haynes MR, Palasis S, et al. Regionally specific cortical thinning in children with sickle cell disease. Cereb Cortex 2009; 19:1549-56

7. Chen R, Pawlak MA, Flynn TB, et al. Brain morphometry and intelligence quotient measurements in children with sickle cell disease. $J$ Dev Behav Pediatr 2009;30:509-17

8. Wang W, Enos L, Gallagher D, et al. Neuropsychologic performance in school-aged children with sickle cell disease: a report from the Cooperative Study of Sickle Cell Disease. J Pediatr 2001;139:391-97

9. Arkuszewski M, Krejza J, Chen R, et al. Sickle cell anemia: intracranial stenosis and silent cerebral infarcts in children with low risk of stroke. Adv Med Sci 2014;59:108-13

10. Evans AC, for the Brain Development Cooperative Group. The NIH MRI Study of Normal Brain Development. Neuroimage 2006; 30:184-202

11. Brain Development Cooperative Group. Total and regional brain volumes in a population-based normative sample from 4 to 18 years: the NIH MRI Study of Normal Brain Development. Cereb Cortex 2012;22:1-12

12. Smith SM. Fast robust automated brain extraction. Hum Brain Mapp 2002;17:143-55

13. Ashburner J, Friston KJ. Unified segmentation. Neuroimage 2005; 26:839-51

14. Tzourio-Mazoyer N, Landeau B, Papathanassiou D, et al. Automated anatomical labeling of activations in SPM using a macroscopic anatomical parcellation of the MNI MRI single-subject brain. Neuroimage 2002;15:273-89

15. Tzarouchi LC, Astrakas LG, Xydis V, et al. Age-related grey matter changes in preterm infants: an MRI study. Neuroimage 2009; 47:1148-53

16. Yamashita K, Yoshiura T, Hiwatashi A, et al. Volumetric asymmetry and differential aging effect of the human caudate nucleus in normal individuals: a prospective MR imaging study. J Neuroimaging 2011;21:34-37

17. Widjaja E, Yeung R, Geibprasert S, et al. Longitudinal brain volumes in children with intractable partial seizures. Pediatr Neurol 2010; 42:315-19

18. Tzarouchi LC, Astrakas LG, Zikou A, et al. Periventricular leukomalacia in preterm children: assessment of grey and white matter and cerebrospinal fluid changes by MRI. Pediatr Radiol 2009;39:1327-32

19. Singer JD, Willett JB. Applied Longitudinal Data Analysis: Modeling Change and Event Occurrence. Oxford: Oxford University Press; 2003

20. Lonergan GJ, Cline B, Abbondanzo SL. Sickle cell anemia. Radiographics 2001;20:971-94

21. Giedd JN, Blumenthal J, Jeffries NO, et al. Brain development during childhood and adolescence: a longitudinal MRI study. Nat Neurosci 1999;2:861-63

22. Matsuzawa J, Matsui M, Konishi T, et al. Age-related volumetric changes of brain gray and white matter in healthy infants and children. Cereb Cortex 2001;11:335-42

23. Sowell ER, Trauner DA, Gamst A, et al. Development of cortical and subcortical brain structures in childhood and adolescence: a structural MRI study. Dev Med Child Neurol 2002;44:4-16

24. Lenroot RK, Giedd JN. Brain development in children and adolescents: insights from anatomical magnetic resonance imaging. Neurosci Biobehav Rev 2006;30:718-29

25. Paus T. Mapping brain maturation and cognitive development during adolescence. Trends Cogn Sci 2005;9:60-68

26. Gould E, Reeves AJ, Graziano MS, et al. Neurogenesis in the neocortex of adult primates. Science 1999;286:548-52

27. Vayo MM, Lipowsky HH, Karp N, et al. A model of microvascular oxygen transport in sickle cell disease. Microvasc Res 1985;30: 195-206

28. Eaton WA, Hofrichter J. Hemoglobin S gelation and sickle cell disease. Blood 1987;70:1245-66

29. Christoph GW, Hofrichter J, Eaton WA. Understanding the shape of sickled red cells. Biophys J 2005;88:1371-76

30. Prohovnik I, Hurlet-Jensen A, Adams R, et al. Hemodynamic etiology of elevated flow velocity and stroke in sickle-cell disease. J Cereb Blood Flow Metab 2009;29:803-10

31. Nath KA, Katusic ZS, Gladwin MT. The perfusion paradox and vascular instability in sickle cell disease. Microcirculation 2004;11: $179-93$

32. van den Tweel XW, Nederveen AJ, Majoie CB, et al. Cerebral blood flow measurement in children with sickle cell disease using continuous arterial spin labeling at 3.0-Tesla MRI. Stroke 2009;40:795-800

33. Hyder F, Shulman RG, Rothman DL. A model for the regulation of cerebral oxygen delivery. J Appl Physiol (1985) 1998;85:554-64

34. Kato GJ, Hebbel RP, Steinberg MH, et al. Vasculopathy in sickle cell disease: biology, pathophysiology, genetics, translational medicine, and new research directions. Am J Hematol 2009;84:618-25

35. Gogtay N, Giedd JN, Lusk L, et al. Dynamic mapping of human cortical development during childhood through early adulthood. Proc Natl Acad Sci U S A 2004;101:8174-79

36. Balci A, Karazincir S, Beyoglu Y, et al. Quantitative brain diffusiontensor MRI findings in patients with sickle cell disease. AJR Am J Roentgenol 2012;198:1167-74

37. Sun B, Brown RC, Hayes L, et al. White matter damage in asymptomatic patients with sickle cell anemia: screening with diffusion tensor imaging. AJNR Am J Neuroradiol 2012;33:2043-49

38. Feldman HM, Yeatman JD, Lee ES, et al. Diffusion tensor imaging: a review for pediatric researchers and clinicians. J Dev Behav Pediatr 2010;31:346-56 